PROCEEDINGS OF THE

AMERICAN MATHEMATICAL SOCIETY

Volume 128, Number 5, Pages 1415-1418

S 0002-9939(99)05233-8

Article electronically published on October 5, 1999

\title{
HILBERT SPACE IDEMPOTENTS AND INVOLUTIONS
}

\author{
DON BUCKHOLTZ
}

(Communicated by Palle E. T. Jorgensen)

\begin{abstract}
Norms of idempotents, involutions, and the Hermitian and skewHermitian parts of involutions are shown to be elementary trigonometric functions of an angle between two subspaces of Hilbert space. When the spaces involved are nontrivial, the norm of a linear idempotent is the cosecant of the angle between its range and kernel; the norm of a linear involution is the cotangent of half the angle between the involution's eigenspaces.
\end{abstract}

A bounded linear idempotent $M$ on a Hilbert space $H$ induces a splitting of $H$ into the direct sum of two subspaces: the range $R$ of $M$ and the kernel $K$. (Throughout this paper the term "subspace" always means a closed linear manifold. That $R$ is a subspace follows from the fact that it is the kernel of the bounded idempotent $I-M$.) The eigenspaces of a linear involution $V$ belonging to the eigenvalues 1 and -1 are the range and kernel of the associated idempotent, $M=$ $(I+V) / 2$. In this paper we study relations between these operators, the orthogonal projections $P_{R}$ and $P_{K}$ of $H$ onto $R$ and $K$, and the angle between the subspaces $R$ and $K$. Our principal results are contained in the following theorems.

Theorem 1. Let $R$ and $K$ be subspaces of a Hilbert space $H$, and let $P_{R}$ and $P_{K}$ denote the orthogonal projections of $H$ onto these subspaces. The following are equivalent:

(i) $H$ is the direct sum of $R$ and $K$.

(ii) There exists a bounded linear idempotent $M$ with range $R$ and kernel $K$.

(iii) The operator $P_{R}-P_{K}$ is invertible.

(iv) $\left\|P_{R}+P_{K}-I\right\|<1$.

The angle between two nontrivial subspaces $R$ and $K$ whose intersection is $\{0\}$ is the number $\theta$ in $[0, \pi / 2]$ whose cosine equals $\sup \{|(r, k)|: r \in R, k \in K,\|r\|=\|k\|=$ $1\}$. In view of $(r, k)=\left(P_{R} r, P_{K} k\right)=\left(r, P_{R} P_{K} k\right)$, we have $\cos (\theta)=\left\|P_{R} P_{K}\right\|=$ $\left\|P_{K} P_{R}\right\|$. See [2] for a discussion of the angle between subspaces whose intersection is nontrivial.

Throughout this paper the term idempotent is used to mean a linear transformation $M$ satisfying $M^{2}=M$, and involution is used to mean a linear transformation $V$ satisfying $V^{2}=I$. Neither is necessarily bounded, and unbounded examples exist on every infinite dimensional Hilbert space. A self-adjoint idempotent is an orthogonal projection.

Received by the editors October 25, 1996 and, in revised form, July 2, 1998.

1991 Mathematics Subject Classification. Primary 46C05; Secondary 47A05, 47A30.

(C)2000 American Mathematical Society 
Theorem 2. Suppose that the Hilbert space $H$ is the direct sum of proper subspaces $R$ and $K$. Let $\theta$ be the angle between $R$ and $K$, let $M$ be the idempotent with range $R$ and kernel $K$, and let $V=2 M-I$ be the associated involution. Then

(i) $\|M\|=\|I-M\|=\left\|I-M^{*}\right\|=\left\|M^{*}\right\|=\csc (\theta)$,

(ii) $\left\|M+M^{*}-I\right\|=\csc (\theta)$,

(iii) $\left\|M-M^{*}\right\|=\cot (\theta)$, and

(iv) $\|V\|=\cot (\theta / 2)$.

Proof of Theorem 1. If (i) holds, then every $x \in H$ has a unique representation of the form $x=r+k, r \in R, k \in K$. Define $M$ by $M x=r$. Clearly $M$ is an idempotent. We use the Closed Graph Theorem [3, prob. 58] to show that $M$ is bounded. Let $\langle x, y\rangle$ be a limit point of the graph of $M$ and $\left\{x_{n}\right\}$ a sequence of points such that $\lim \left\langle x_{n}, M x_{n}\right\rangle=\langle x, y\rangle$. What needs to be shown is that $y=M x$. For each positive integer $n$ let $x_{n}=r_{n}+k_{n}, r_{n} \in R, k_{n} \in K$. Then $\left\langle x_{n}, M x_{n}\right\rangle=\left\langle r_{n}+k_{n}, r_{n}\right\rangle$ and we have $\lim \left(r_{n}+k_{n}\right)=x$ and $\lim \left(r_{n}\right)=y$. Therefore we have $y \in R$ and $\lim k_{n}=x-y \in K$ since $R$ and $K$ are closed. Consequently, $M(x-y)=0$ and $M y=y$, since an idempotent is the identity on its range. Therefore $M x=y$, and (i) implies (ii).

The easy way to prove that (ii) $\Rightarrow$ (iiii) is to exhibit an inverse for $P_{R}-P_{K}$. We shall make frequent use of the fact that, if $M$ is an idempotent with range $R$ and kernel $K$, then $M^{*}$ is an idempotent with range $K^{\perp}$ and kernel $R^{\perp}$. Additionally, $I-M$ is idempotent with range $K$ and kernel $R$. Finally, $I-M^{*}$ is an idempotent with range $R^{\perp}$ and kernel $K^{\perp}$. Therefore

$$
P_{R} M=M, \quad P_{K} M^{*}=0, \quad P_{K}(I-M)=I-M, \quad \text { and } \quad P_{R}\left(I-M^{*}\right)=0 .
$$

Combining these results yields $\left(P_{R}-P_{K}\right)\left(M+M^{*}-I\right)=I$. These operators are Hermitian; by taking adjoints we get $\left(P_{R}-P_{K}\right)\left(M+M^{*}-I\right)=I$. Therefore $\left(P_{R}-P_{K}\right)^{-1}=M+M^{*}-I$. This result was obtained in [1], and is the nice special case of each of the more general inversion formulas

$$
\left(\alpha P_{R}+\beta P_{K}\right)^{-1}=(1 / \beta)\left(I-M-M^{*}\right)+(1 / \alpha+1 / \beta) M^{*} M
$$

and

$$
\left(\alpha M+\beta\left(I-M^{*}\right)\right)^{-1}=(1 / \alpha) P_{R}+(1 / \beta) P_{K}-(1 / \alpha+1 / \beta) P_{K} P_{R},
$$

whose proofs are similar.

To establish the implication (iii) $\Rightarrow$ (iv) we use the identity

$$
\left\|\left(P_{R}-P_{K}\right) x\right\|^{2}+\left\|\left(P_{R}+P_{K}-I\right) x\right\|^{2}=\|x\|^{2} .
$$

Set $\mu=\inf \left\{\left\|\left(P_{R}-P_{K}\right) x\right\|:\|x\|=1\right\}$. We have $\mu^{2}+\left\|P_{R}+P_{K}-I\right\|^{2}=1$. Since $P_{R}-P_{K}$ is invertible, $\mu>0$ and (iv) follows.

It remains to show that (iv) implies (i). Certainly (iv) implies the invertibility of $P_{R}+P_{K}$, and the identity $x=P_{R}\left(P_{R}+P_{K}\right)^{-1} x+P_{K}\left(P_{R}+P_{K}\right)^{-1} x$ insures that $H=R+K$. Note that (iv) also requires $R$ and $K$ to be disjoint except for 0 . Therefore $H$ is the direct sum of $R$ and $K$, and the proof of Theorem 1 is complete.

While it is too much to claim that Theorem 2 is geometrically obvious, the theorem is certainly geometrically plausible. Let $\mathcal{P}_{\theta}$ be a parallelogram with acute angle $\theta$. The maximum value of the side/diagonal ratio is $\csc (\theta)$, achieved when the side is the longer side, the diagonal is the shorter diagonal, and that diagonal is an altitude. Considerably less obvious is the fact that the maximum value of the diagonal/diagonal ratio is $\cot (\theta / 2)$, achieved when $\mathcal{P}_{\theta}$ is equilateral. 
In $H$ the parallelograms to consider are those with vertices at $0, M x, 2 M x-x$, and $M x-x$. The vectors $M x$ and $M x-x$ belong to $R$ and $K$ respectively, while $x$ and $V x=2 M x-x$ are the diagonal vectors of this parallelogram. Observe that $\|M x\| /\|x\|$ is a side to diagonal ratio and $\|V x\| /\|x\|$ is a diagonal to diagonal ratio. This puts (i) and (iv) of Theorem 2 in the category of reasonable conjectures.

The condition that the diagonal vector $x$ be an altitude makes sense: it simply requires that $x$ belong to $K^{\perp}$. If restricting the operators $M+M^{*}-I$ and $M-M^{*}$ to $K^{\perp}$ does not diminish their norms, then parts (ii) and (iii) are equally reasonable conjectures. On $K^{\perp}, M+M^{*}-I$ agrees with $M$ and the operator $M-M^{*}$ agrees with $M-I$. The norm for $M-M^{*}$ follows from the Pythagorean theorem and the trigonometric identity $\cot ^{2}(\theta)=\csc ^{2}(\theta)-1$.

Lemma. Let $M$ be a bounded linear idempotent with range $R$ and kernel $K$. If $M \neq 0$, then

$$
\|M\|=\left\{1-\left\|P_{K} P_{R}\right\|^{2}\right\}^{-1 / 2},
$$

where $P_{R}$ and $P_{K}$ denote the orthogonal projections onto $R$ and $K$.

Proof. For $x \in H$ write $x=x_{1}+x_{2}$, where $x_{1}=P_{K} x$ and $x_{2}=\left(I-P_{K}\right) x$. Then $M x=M x_{2}$ and $\left\|x_{2}\right\| \leq\|x\|$. Consequently the norm of $M$ is the same as the norm of the restriction of $M$ to $K^{\perp}$. For $x \in K^{\perp}$ write $M x=x+(M x-x)$ and observe that $(M x-x)$ is the orthogonal projection of $M x$ onto $K$. Therefore $M x-x=P_{K} M x=P_{K} P_{R} M x$, since $M x \in R$. From $x \in K^{\perp}$ and $(M x-x) \in K$ we obtain $\|M x\|^{2}=\|x\|^{2}+\|M x-x\|^{2}=\|x\|^{2}+\left\|P_{K} P_{R} M x\right\|^{2}$. Division by $\|M x\|^{2}$ and some manipulation yields the identity

$$
\|M x\| /\|x\|=\left\{1-\left(\left\|P_{K} P_{R} M x\right\| /\|M x\|\right)^{2}\right\}^{-1 / 2} .
$$

It follows from this that $\|M\| \leq\left\{1-\left\|P_{K} P_{R}\right\|^{2}\right\}^{-1 / 2}$. To obtain equality rather than inequality, two observations are needed: restricting the operator $M$ to $K^{\perp}$ does not change its range, and restricting the operator $P_{K} P_{R}$ to $R$ does not diminish its norm. This completes the proof.

Proof of Theorem 2, From our lemma we have

$$
\|M\|=\left\{1-\left\|P_{K} P_{R}\right\|^{2}\right\}^{-1 / 2}=\left\{1-\cos ^{2}(\theta)\right\}^{-1 / 2}=\csc (\theta) .
$$

The order of the operators $P_{R}$ and $P_{K}$ is unimportant; $P_{K} P_{R}$ and $P_{R} P_{K}$ have the same norm because they are adjoints. Since $I-M$ is an idempotent with range $K$ and kernel $R$ we also have the corresponding result that $\|I-M\|=\|M\|=\csc (\theta)$. Because they are the adjoints of $I-M$ and $M$, we also have $\left\|I-M^{*}\right\|=\left\|M^{*}\right\|=$ $\csc (\theta) . \quad M^{*}$ is an idempotent with range $K^{\perp}$ and kernel $R^{\perp}$, and $I-M^{*}$ is an idempotent with range $R^{\perp}$ and kernel $K^{\perp}$. Either of these implies the result that

$$
\left\|P_{K} P_{R}\right\|=\left\|\left(I-P_{R}\right)\left(I-P_{K}\right)\right\| \text {. }
$$

That is, the angle between $R^{\perp}$ and $K^{\perp}$ is the same as the angle between $R$ and $K$.

The proof of (ii) is easy but slightly devious. Set $S=M+M^{*}-I$. We have already established that the restriction of $M$ to $K^{\perp}$ has the same norm as $M$. On this subspace $M^{*}-I$ vanishes and $S$ agrees with $M$. Therefore $\|S\| \geq \csc (\theta)$.

For $x \in H$ write $x=x_{1}+x_{2}$, where $x_{1}=P_{K} x$ and $x_{2}=\left(I-P_{K}\right) x$. Then

$$
S x_{1}=\left(M^{*}-I\right) x_{1} \in R^{\perp} \quad \text { and } \quad S x_{2}=M x_{2} \in R .
$$


Since $S x_{1}$ and $S x_{2}$ belong to orthogonal subspaces, we have

$$
\|S x\|^{2}=\left\|\left(M^{*}-I\right) x_{1}\right\|^{2}+\left\|M x_{2}\right\|^{2} \leq \csc ^{2}(\theta)\left\{\left\|x_{1}\right\|^{2}+\left\|x_{2}\right\|^{2}\right\}=\csc ^{2}(\theta)\|x\|^{2} .
$$

Therefore $\|S\| \leq \csc (\theta)$, and the proof of (ii) is complete.

Part (iii) is an easy consequence of (ii) and the identity

$$
\left\|\left(M+M^{*}-I\right) x\right\|^{2}=\|x\|^{2}+\left\|\left(M-M^{*}\right) x\right\|^{2} .
$$

We now deal with (iv). From the triangle inequality and $V=2 M-I$ we have

$$
\|V\| \leq\left\|M+M^{*}-I\right\|+\left\|M-M^{*}\right\|=\csc (\theta)+\cot (\theta)=\cot (\theta / 2) .
$$

Choose $r \in R$ and $k \in K$ such that $\|r\|=\|k\|=1$. Define $\varphi$ in $[0, \pi / 2]$ by $|(r, k)|=\cos (\varphi)$, and let $\alpha$ be a complex number of absolute value 1 chosen to make the inner product $(\alpha r, k)$ real and nonnegative. Set $x=\alpha r-k$. Then we have $V x=(2 M-I) x=\alpha r+k$. We also have

$$
\begin{aligned}
\|V x\|^{2} & =(\alpha r+k, \alpha r+k)=\|\alpha r\|^{2}+\|k\|^{2}+(\alpha r, k)+(k, \alpha r) \\
& =2+2(\alpha r, k)=2+2|(r, k)|=2+2 \cos (\varphi) .
\end{aligned}
$$

Similarly, $\|x\|^{2}=2-2(\alpha r, k)=2-2 \cos (\varphi)$. Therefore $\|V\| \geq\|V x\| /\|x\|=$ $\cot (\varphi / 2)$. Since $\theta$ is the infimum of the set of values for $\varphi$, we have $\|V\| \geq \cot (\theta / 2)$. This establishes (iv).

Unbounded idempotents. Provided that the space is not finite dimensional, unbounded idempotents exist and are relatively easy to construct in any normed linear space. Given an infinite dimensional normed linear space $H$, let $B$ be a Hamel basis whose members have norm 1 . Let $E=\left\{\beta_{1}, \beta_{2}, \beta_{3}, \ldots\right\}$ be a countably infinite subset of $B$. We define a linear operator $M$ on $H$ by specifying its values on members of $B$. For those vectors $\beta \in B$ which do not belong to $E$, let $M \beta=0$. For each positive integer $n$, let $M \beta_{n}=n \beta_{1}$. Clearly $M$ is unbounded and, because $M$ is idempotent on $B, M$ is idempotent on $H$. The range of $M$ is the one-dimensional subspace spanned by $\beta_{1}$. The kernel of $M$ is a linear manifold whose closure is all of $H$. To see this, note that the terms in the sequence $\left\{\beta_{1}-\beta_{n} / n\right\}$ belong to the kernel and have $\beta_{1}$ as their limit. Therefore the range of $M$ is contained in the closure of its kernel. Since $H$ is the sum of the range and kernel of $M$, it follows that $H$ is the closure of the kernel of $M$.

\section{REFERENCES}

1. Don Buckholtz, Inverting the difference of Hilbert space projections, Amer. Math. Monthly 104 (1997), 60-61. MR 98a:47002

2. Frank Deutsch, von Neumann's alternating method: the rate of convergence, "Approximation Theory IV", C. Chui, L. Schumaker, J. Ward, eds., Academic Press, New York, London, 1983, 427-434. MR 85m:41040

3. Paul R. Halmos, A Hilbert space problem book, 2nd edition, Springer-Verlag, New York, 1982. MR 84e:47001

Department of Mathematics, University of Kentucky, Lexington, Kentucky 40506

E-mail address: mat236@ukcc.uky.edu 